\title{
Kinetic Model to Predict the Absorption of Nasally Applied Drugs from in Vitro Transcellular Permeability of Drugs
}

\author{
Tomoyuki Furubayashi, ${ }^{*}, a$ Akiko Kamaguchi, ${ }^{a}$ Kazushi Kawaharada, ${ }^{b}$ Yoshie Masaoka, ${ }^{b}$ \\ Makoto KataOKa, ${ }^{b}$ Shinji Yamashita, ${ }^{b}$ Yutaka Higashi, ${ }^{a}$ and Toshiyasu SAKAnE ${ }^{a}$ \\ ${ }^{a}$ School of Pharmacy, Shujitsu University; 1-6-1 Nishigawara, Okayama 703-8516, Japan: and ${ }^{b}$ Faculty of \\ Pharmaceutical Sciences, Setsunan University; 45-1 Nagaotoge-cho, Hirakata, Osaka 573-0101, Japan. \\ Received November 2, 2006; accepted February 2, 2007; published online February 6, 2007
}

The purpose of this study is to propose a kinetic model to predict the absorption of nasally applied drugs from their permeability to the Caco-2 monolayer $\left(\mathrm{P}_{\mathrm{Caco-2}}\right)$. Since a drug applied to the nose in an in vivo physiologic condition is translocated to the gastrointestinal (GI) tract by coordinated beats of cilia (mucociliary clearance, MC), the drug undergoes absorption both from the nasal cavity and from the GI tract. The detailed MC of the rat was examined, using inulin as a marker of the applied solution. Inulin disappeared monoexponentially from the nasal cavity, indicating that the MC can be assumed to follow first-order kinetics. From the disappearance of inulin, the first order rate constant for $\mathrm{MC}\left(k_{\mathrm{MC}}\right)$ was calculated as $0.0145 \mathrm{~min}^{-1}$. In the proposed kinetic model, the fractional absorption of the drug following nasal application is predicted as the sum of $F_{\mathrm{NC}}$ (fractional absorption from the nasal cavity) and $F_{\mathrm{GI}}$ (fractional absorption from the GI tract), both of which are estimated indirectly from $P_{\mathrm{Caco-2}} . F_{\mathrm{NC}}$ is calculated according to the equation, $k_{\mathrm{a}} /\left(k_{\mathrm{a}}+k_{\mathrm{MC}}\right)$, where $k_{\mathrm{a}}$ is the absorption rate constant. Nasal drug absorption is assumed to follow first order kinetics. The $k_{\mathrm{a}}$ of four drugs was initially calculated from $k_{\mathrm{MC}}$ and their $F_{\mathrm{NC}}$; thereafter, the linear relationship between $\boldsymbol{k}_{\mathrm{a}}$ and $\boldsymbol{P}_{\mathrm{Caco-2}}$, from which $\boldsymbol{k}_{\mathrm{a}}$ is predicted, was determined. $F_{\mathrm{GI}}$ is calculated as $F_{p . o}\left(1-F_{\mathrm{NC}}\right)$, where $F_{p . o .}$ is fractional absorption after oral administration. $F_{\text {p.o. }}$ was predicted from the previously determined sigmoid curve between $\boldsymbol{F}_{\text {p.o. }}$ and $\boldsymbol{P}_{\mathrm{Cac0-2} 2}$. The proposed kinetic model is the first estimation system for nasal drug absorption based on drug disposition after nasal application and is useful for the development of nasal dosage forms.

Key words nasal absorption; prediction; Caco-2 monolayer; mucociliary clearance

In the last few decades, nasal administration has received a great deal of attention as a rationale for the systemic delivery of many drugs. ${ }^{1)}$ The range of compounds investigated for possible nasal application varies greatly from very lipophilic drugs to polar, hydrophilic molecules including peptides and proteins. $\left.{ }^{2}\right)$ The relatively high permeability of the nasal epithelium, its high vascularization and the avoidance of hepatic first-pass metabolism makes nasal application a promising alternative, especially for drugs exhibiting high metabolism in the intestine and/or liver.

Oral drug absorption can be estimated from in vitro transepithelial transport across the Caco-2 monolayer with various systems. ${ }^{3-5)}$ These systems are responsible for screening of huge number of new drug candidates which are synthesized through combinatorial chemistry and pharmacologically screened with an in vitro high throughput system. Although nasal administration has been considered important as an alternative to oral application, as mentioned above, no prediction system has been developed so far. A prediction system would greatly help the development of nasal medications.

Some respiratory epithelial cells possess cilia on their surface. The cilia beat in a coordinated fashion to transport the mucous layer, which covers the surface of the epithelium, to the nasopharynx, where it is swallowed. ${ }^{6-8)}$ The combined action of the mucus layer and cilia is called mucociliary clearance (MC). It is an important nonspecific defense mechanism of the respiratory tract to protect the body against noxious inhaled materials. Due to MC, drugs applied to the nasal cavity are translocated to the nasopharynx and, thereafter, to the gastrointestinal (GI) tract, together with the mucus layer. Some fraction of the nasally-administered drug undergoes absorption from the GI tract. In order to develop a predictive system for fractional drug absorption after nasal application, the kinetic characteristics of mucociliary clearance must be clarified and correctly combined in the kinetic model.

In the previous manuscript, ${ }^{9}$ ) five non-degradable drugs were selected as model drugs and their fractional absorption following nasal and oral application, and their permeability to the Caco-2 monolayer $\left(P_{\mathrm{Caco-} 2}\right)$ were examined. The methods for the calculation of fractional absorption from the nasal cavity and from the GI tract after nasal application were also described. The relationship between fractional absorption and $P_{\text {Caco-2 }}$ was discussed, and the feasibility to predict drug absorption following nasal administration from $P_{\mathrm{Caco}-2}$ was indicated. The first aim of this research is to clarify the details of MC. For this purpose, the surgical operation reported by Hirai et al. $^{10)}$ was not done on the esophagus and trachea, and the animal was kept conscious for as long as possible during the animal study. Based on the information on $\mathrm{MC}$, the second aim is to propose a kinetic model to predict drug absorption following nasal application to rats from $P_{\mathrm{Caco}-2}$. Various fractional absorptions reported previously were utilized in this study. From these values, the correlation between the kinetic parameter and $P_{\mathrm{Caco}-2}$ was determined and applied for the prediction of total drug absorption after nasal administration.

Theory and Kinetic Model Drugs applied nasally undergo absorption both from the nasal cavity and from the GI tract. Total fractional absorption following nasal drug application $\left(F_{\mathrm{n}}\right)$ is considered the sum of two fractional absorptions, $F_{\mathrm{NC}}$ and $F_{\mathrm{GI}} \cdot F_{\mathrm{NC}}$ is the fraction of the drug absorbed from the nasal cavity and $F_{\mathrm{GI}}$ is that from the GI tract following nasal drug application. 


$$
F_{\mathrm{n}}=F_{\mathrm{NC}}+F_{\mathrm{GI}}
$$

To simplify the prediction model, no degradation and metabolism of the drug in the nasal cavity was taken into consideration in this study. The model drugs had been selected in the previous report ${ }^{9)}$ as not metabolizing and degrading in the nasal cavity.

Prediction of Fractional Absorption from the Nasal Cavity The drug is eliminated from the nasal cavity both by absorption into systemic circulation and by mucociliary translocation. The assumptions listed below were made for the prediction of $F_{\mathrm{NC}}$.

Assumption 1: The same value of $k_{\mathrm{MC}}$, which is a first order rate constant for $\mathrm{MC}$, can be applied to all drugs. Since the drug is translocated by $\mathrm{MC}$, together with the dosing solution, the mucociliary translocation of the drug is independent of the physicochemical properties of the drug.

Assumption 2: Drug absorption from the nasal cavity is assumed to follow first order kinetics with a rate constant, $k_{\mathrm{a}}$.

Assumption 3: There exists a proportional relationship between $k_{\mathrm{a}}$ and $P_{\mathrm{Caco}-2}$.

The data from the study on the disappearance of inulin from the nasal cavity showed that MC follows first order kinetics with the rate constant, $k_{\mathrm{MC}}$. Based on Assumption 2, $F_{\mathrm{NC}}$ can be calculated according to the following equation.

$$
F_{\mathrm{NC}}=\frac{k_{\mathrm{a}}}{k_{\mathrm{a}}+k_{\mathrm{MC}}}
$$

To predict $F_{\mathrm{NC}}$ of the drug, $k_{\mathrm{a}}$ is initially estimated from the proportional relationship between $k_{\mathrm{a}}$ and $P_{\mathrm{Caco}-2}$ (Assumption 3 ) and then $\mathrm{F}_{\mathrm{NC}}$ is calculated according to Eq. 2. A correlation equation between $k_{\mathrm{a}}$ and $P_{\mathrm{Caco}-2}$ was determined in this report using data from four drugs.

Prediction of Fractional Absorption from GI Tract The prediction of $F_{\mathrm{GI}}$ is very simple. A non-degradable drug is translocated to the GI tract at the fraction of $1-F_{\mathrm{NC}}$ and is absorbed from the GI tract at the fraction of $F_{p . o .}$. Consequently, $F_{\mathrm{GI}}$ can be calculated as follows.

$$
F_{\mathrm{GI}}=F_{p . o .}\left(1-F_{\mathrm{NC}}\right)
$$

Substitution of Eqs. 2 and 3 into Eq. 1 results in the following equation.

$$
F_{\mathrm{n}}=\frac{k_{\mathrm{a}}+F_{p . o .} \cdot k_{\mathrm{MC}}}{k_{\mathrm{a}}+k_{\mathrm{MC}}}
$$

Equation 4 indicates that the total fractional absorption of the drug after nasal application can be calculated from three parameters. $k_{\mathrm{a}}$ and $F_{\text {p.o. }}$ are predicted from $P_{\mathrm{Caco}-2}$, while $k_{\mathrm{MC}}$ is a constant irrespective of the difference in the physicochemical properties of drugs.

\section{MATERIALS AND METHODS}

Materials Inulin-methoxy, [methoxy- ${ }^{14} \mathrm{C}$ ], is a product of American Radiolabeled Chemicals Inc. (St. Louis, MO, U.S.A.) and was purchased from the Japan Radioisotope As- sociation. All other chemicals were of reagent grade and commercially available.

Disappearance of Inulin from the Rat Nasal Cavity All animal studies were previously approved by the Committee of the Animal Care of the University and conducted under the Guidelines. Male Wistar rats (B.W. 200-260 g) were used in animal experiments.

Under light ether anesthesia, $5 \mu$ l of inulin solution was instilled at $1 \mathrm{~cm}$ depth from the nostril with a microsyringe. The rat was kept in an animal cage thereafter to recover from the anesthesia. The animal usually regained complete consciousness 5-10 min after instillation. The trachea and esophagus were canulated as described by Hirai et al. ${ }^{10)} 0$, $30,60,120$ and $180 \mathrm{~min}$ after instillation under ether anesthesia. The nasal cavity of the rat was washed five times through the cannula with $2 \mathrm{ml}$ of physiological saline. The washings were collected together to determine the total volume. Soon after washing, the rat was decapitated. The nasal mucosal tissue was dissected.

Nasal washings $(100 \mu \mathrm{l})$ were transferred to a counting vial and treated with $0.5 \mathrm{ml}$ of Soluene 350 (Perkin-Elmer, Wellesley, MA, U.S.A.). The nasal tissue was dissolved in $1 \mathrm{ml}$ of Soluene 350. Radioactivity in the samples was determined with a liquid scintillation counter, LSC3500 (Aloka, Tokyo, Japan) after adding $10 \mathrm{ml}$ of Clearsol II (Nacalai Tesque, Kyoto, Japan). The amount of inulin in the nasal cavity was calculated from the total radioactivity in washings and the nasal tissue, and normalized with the total applied activity.

\section{RESULTS AND DISCUSSION}

Disappearance of Inulin from the Nasal Cavity Figure 1 shows the elimination of inulin from the nasal cavity as a function of time. The disappearance of inulin from the nasal cavity was rapid. Inulin was eliminated $180 \mathrm{~min}$ after instillation. The semilogarithmic plot of the curve was almost linear. Akaike's information criteria (AIC) calculated with a computer program of nonlinear regression analysis, MULTI, ${ }^{11)}$ clarified that a monoexponential equation is better for the elimination of inulin than a biexponential equation. The rate constant (disappearance rate constant, $k_{\text {dis }}$ ) was calculated with MULTI. The dashed line shown in Fig. 1 is the result and $k_{\text {dis }}$ is obtained as $0.0152 \mathrm{~min}^{-1}$. Consequently, the disappearance half life of inulin from the nasal cavity is $37.1 \mathrm{~min}$.

Hardy et al. ${ }^{12)}$ reported the MC of humans. Radioactive albumin instilled or sprayed nasally into human subjects was detected by $\gamma$-camera. Examination of the disappearance time courses of albumin clarified that albumin in the human nasal cavity was eliminated monoexponentially. Since the molecular size of albumin is so large, the contribution of absorption to the disappearance of albumin from the nasal cavity is negligible. This indicates that there may exist no species difference in MC irrespective of the large difference in the structure and anatomy of the nasal cavity.

Determination of the First Order Rate Constant for MC If drug absorption from the nasal cavity is assumed to follow first order kinetics, mucociliary translocation of the drug also follows first order kinetics. Although inulin was poorly absorbable and used as a marker of the dosing solution, $F_{\mathrm{NC}}$ of inulin was 0.046 . Consequently, both the translo- 

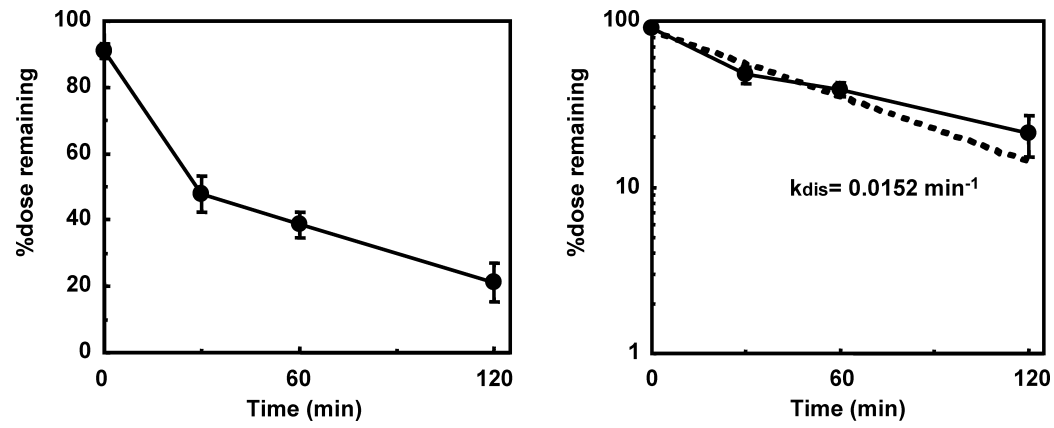

Fig. 1. Disappearance of Inulin from the Rat Nasal Cavity

AICs calculated with the nonlinear regression program are 29.3 for a monoexponential equation and 30.7 for a biexponential equation, suggesting that the monoexponential equation is more suitable for the disappearance of inulin.

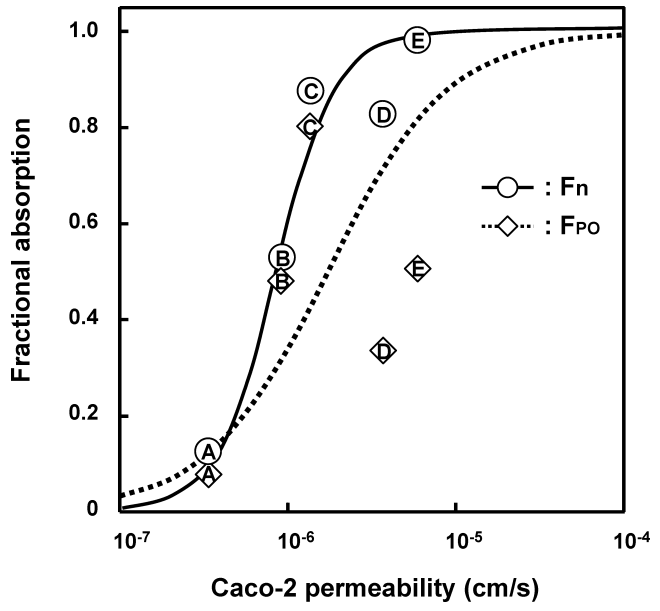

Fig. 2. The Relationship between Fractional Absorption after Oral Drug Administration and Permeability to Caco-2 Monolayer

A: inulin, B: mannitol, C: acyclovir, D: sulfanilic acid, E: methotrexate. The fitted lines of $F_{\mathrm{n}}$ and $F_{\mathrm{NC}}$ were obtained from a Hill's sigmoidal equation.

cation of the drug and absorption contributed to the disappearance of inulin from the nasal cavity. The calculation shown in appendix 1 indicates that $k_{\mathrm{MC}}$ is $0.0145 \mathrm{~min}^{-1}$. The contribution of absorption and translocation to the disappearance of inulin from the nasal cavity is $4.6 \%$ and $95.4 \%$, respectively.

Relationship of $\boldsymbol{F}_{\text {p.o. }}$ and $\boldsymbol{F}_{\mathbf{n}}$ to $\boldsymbol{P}_{\text {Caco-2 }}$ Figure 2 shows the sigmoid curve between $F_{\text {p.o. }}$ and $P_{\text {Caco-2 }}$. Since $F_{\text {p.o. }}$ of the model drugs was scattered from the sigmoid curve, the curves were obtained assuming a similar shape and location to those reported by Artursson and Karlsson. ${ }^{3)}$ More data are necessary to identify the precise relationship between $F_{p . o}$. and $P_{\text {Caco-2 }}$. The relation between $F_{\mathrm{n}}$ and $P_{\mathrm{Caco}-2}$ is also indicated in Fig. 2. In contrast with $F_{\text {p.o. }}$, the shape of the curve is sigmoid.

Caco-2 is very popular and widely used for in vitro experiments. ${ }^{4)}$ The origin of Caco-2 is the human colon carcinoma $^{13)}$; therefore, it is reasonable to use this cell line for the prediction of oral bioavailability. Recently, Calu-3 cells have been used for the in vitro system of pulmonary and nasal drug absorption. ${ }^{14,15)}$ Calu-3 was derived from human lung carcinoma. Judging from the origin of the cell line, Calu-3 cells was used in the present study. Many studies have been done on the characterization of Caco-2 and much information is available from the literature. Researchers have used

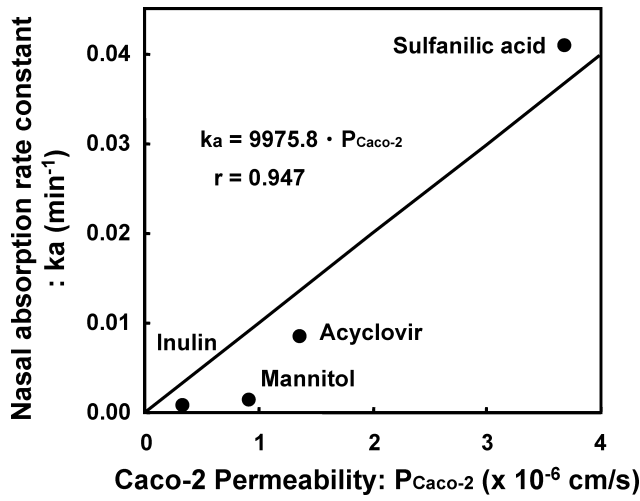

Fig. 3. The Relationship between Absorption Rate Constant and Permeability to Caco-2 Monolayer

Caco-2 for many years. The kinetic model utilizing Caco-2 has many advantages over that using Calu-3, which is why Caco-2 was used in this study. The permeability of drugs to Calu-3 was determined and compared. The results showed no significant differences in drug permeabilities to Caco-2 and Calu-3.

Determination of the Correlation between $\boldsymbol{k}_{\mathrm{a}}$ and $\boldsymbol{P}_{\mathrm{Caco-2}}$ Table 1 lists $P_{\text {Caco-2 }}, F_{\mathrm{n}}, F_{\text {p.o. }}, F_{\mathrm{NC}}$ and $F_{\mathrm{GI}}$ of the model drugs which were previously reported. $\left.{ }^{9}\right)$ From $k_{\mathrm{MC}}\left(0.0145 \mathrm{~min}^{-1}\right)$ and $F_{\mathrm{NC}}$ listed in Table 1 , the $k_{\mathrm{a}}$ of the drugs, except for methotrexate, were calculated according to Eq. 8. The result is also indicated in Table 1. Figure 3 shows the correlation of $k_{\mathrm{a}}$ to $P_{\mathrm{Caco}-2}$. The line in Fig. 3 is expressed as

$$
k_{\mathrm{a}}\left(\min ^{-1}\right)=9975.8 \times P_{\text {Caco-2 }}(\mathrm{cm} / \mathrm{s})
$$

with the correlation coefficient of 0.947 ( $p<0.01$ by Student's $t$-test). Data of methotrexate were excluded from Fig. 3 since the calculation of $k_{\mathrm{a}}$ gives an erroneous result in the case of the drug with almost complete absorption (see appendix 2).

The reliability and precision of the estimation system is dependent on the correlation of $k_{\mathrm{a}}$ with $P_{\mathrm{Caco-2}}$. Equation 5 was determined using the data of four drugs, but these data are likely not sufficient. Since it is not feasible to determine $k_{\mathrm{a}}$ of highly permeable drugs such as methotrexate, the model drugs for this purpose are restricted $\left(5 \times 10^{-7}<P_{\text {Caco-2 }}(\mathrm{cm} / \mathrm{s})<\right.$ $\left.10^{-5}\right)$. A study to improve the reliability of the system is now under investigation.

From Eq. $4, k_{\mathrm{a}}, F_{\text {p.o. }}$ and $k_{\mathrm{MC}}$ are required for the estimation of fractional absorption following nasal drug application. The relationship between $P_{\mathrm{Caco-2}}$ and $F_{\text {p.o. }}$ in human is avail- 
Table 1. In Vitro Transepithelial Permeabilities and in Vivo Pharmacokinetic Parameters of the Model Drugs

\begin{tabular}{|c|c|c|c|c|c|c|}
\hline \multirow{2}{*}{ Compounds } & \multirow{2}{*}{$\begin{array}{l}\text { Caco-2 permeability } \\
P_{\text {Caco-2 }^{a)}}\left(\times 10^{-6} \mathrm{~cm} / \mathrm{s}\right)\end{array}$} & \multicolumn{4}{|c|}{ Fractional absorption } & \multirow{2}{*}{$\begin{array}{c}\begin{array}{c}\text { Nasal absorption } \\
\text { rate constant }\end{array} \\
k_{\mathrm{a}}^{c)}\left(\mathrm{min}^{-1}\right)\end{array}$} \\
\hline & & $F_{\mathrm{n}}^{a)}$ & $F_{p . o .}^{a)}$ & $F_{\mathrm{NC}}^{b)}$ & $F_{\mathrm{GI}}^{b)}$ & \\
\hline Inulin & $0.34 \pm 0.07$ & $0.124 \pm 0.020$ & $0.082 \pm 0.014$ & 0.046 & 0.078 & $0.70 \times 10^{-3}$ \\
\hline Mannitol & $0.92 \pm 0.15$ & $0.529 \pm 0.034$ & $0.484 \pm 0.044$ & 0.087 & 0.442 & $1.38 \times 10^{-3}$ \\
\hline Acyclovir & $1.37 \pm 0.21$ & $0.877 \pm 0.079$ & $0.805 \pm 0.135$ & 0.369 & 0.508 & $8.48 \times 10^{-3}$ \\
\hline Sulfanilic acid & $3.69 \pm 0.40$ & $0.827 \pm 0.090$ & $0.339 \pm 0.024$ & 0.738 & 0.089 & $40.84 \times 10^{-3}$ \\
\hline Methotrexate & $5.95 \pm 0.29$ & $0.982 \pm 0.136$ & $0.508 \pm 0.010$ & 0.963 & 0.019 & - \\
\hline
\end{tabular}

a) $P_{\mathrm{Coc}-2}, F_{\mathrm{n}}$ and $F_{p o}$ were obtained from the data of in vitro Caco-2 study and in vivo animal study. These values are given as the mean \pm S.E. of at least three independent experiments. b) $F_{\mathrm{NC}}$ and $F_{\mathrm{GI}}$ were calculated from $F_{\mathrm{n}}$ and $F_{\text {p.o. }}$ (Furubayashi et al. 2007). c) $k_{\mathrm{a}}$ was obtained from $k_{\mathrm{MC}}\left(0.0145 \mathrm{~min}{ }^{-1}\right)$ and $F_{\mathrm{NC}}$ according to Eq. 8 .

able. ${ }^{3)}$ As mentioned above, $k_{\mathrm{MC}}$ of humans can be calculated from the data reported by Hardy et al. ${ }^{11)}$ In order to estimate $F_{\mathrm{n}}$ in human, $k_{\mathrm{a}}$ is necessary. The accumulation of data on human nasal absorption will provide a system with feasible application to humans.

In conclusion, the proposed kinetic model is the first estimation system for nasal drug absorption based on drug disposition after nasal application and is useful for the development of nasal dosage forms. The system may be applicable to the estimation of nasal absorption in humans if more information on fractional nasal absorption in humans becomes available.

Appendix 1: Calculation of $\boldsymbol{k}_{\mathrm{MC}} k_{\mathrm{dis}}$ of inulin is $0.0152 \mathrm{~min}^{-1}$, equal to the sum of $k_{\mathrm{a}}$ and $k_{\mathrm{MC}}$.

$$
k_{\mathrm{dis}}=k_{\mathrm{a}}+k_{\mathrm{MC}}=0.0152
$$

Since $F_{\mathrm{NC}}$ of inulin is 0.046 , Eq. 2 for inulin is as follows:

$$
F_{\mathrm{NC}}=\frac{k_{\mathrm{a}}}{k_{\mathrm{a}}+k_{\mathrm{MC}}}=0.046
$$

From Eqs. 6 and 7, $k_{\mathrm{MC}}$ was calculated as $0.0145 \mathrm{~min}^{-1}$.

Appendix 2: Calculation of $\boldsymbol{k}_{\mathrm{a}}$ of Model Drugs $k_{\mathrm{a}}$ of the drug was calculated from $k_{\mathrm{MC}}$ and $F_{\mathrm{NC}}$ according to the following equation which is obtained by rearrangement of Eq. 2.

$$
k_{\mathrm{a}}=\frac{F_{\mathrm{NC}} \cdot k_{\mathrm{MC}}}{1-F_{\mathrm{NC}}}
$$

In Eq. $8, k_{\mathrm{MC}}$ is $0.0145 \mathrm{~min}^{-1}$ and the $F_{\mathrm{NC}}$ of each drug listed in Table 1 is used. Since the denominator of Eq. 8 is $1-F_{\mathrm{NC}}$, the calculation of $k_{\mathrm{a}}$ of the drug with $F_{\mathrm{NC}}$ almost equal to 1 is influenced very much by experimental error to give an erroneous result. Consequently, $k_{\mathrm{a}}$ of methotrexate with an $F_{\mathrm{NC}}$ of 0.963, was excluded from Fig. 3.

\section{REFERENCES}

1) Tirucherai G. S., Yang C., Mitra A. K., Expert Opin. Biol. Ther., 1, 49-66 (2001).

2) Illum L., J. Control. Release, 87, 187-198 (2003).

3) Artursson P., Karlsson J., Biochem. Biophys. Res. Commun., 175, $880-885$ (1991).

4) Artursson P., Palm K., Luthman K., Adv. Drug Deliv. Rev., 46, 27-43 (2001).

5) Yamashita S., Tanaka Y., Endoh Y., Taki Y., Sakane T., Nadai T., Sezaki H., Pharm. Res., 16, 486-491 (1997).

6) Illum L., J. Aerosol. Med., 19, 92-99 (2006).

7) Martin E., Schipper N. G. M., Verhoef J. C., Merkus F. W. H. M., $A d v$ Drug Deliv. Rev., 29, 13-38 (1998).

8) Schipper N. G. M., Verhoef J. C., Merkus F. W. H. M., Pharm. Res., 8 807-814 (1991).

9) Furubayashi T., Kamaguchi A., Kawaharada K., Masaoka Y., Kataoka M., Yamashita S., Higashi Y., Sakane T. Biol. Pharm. Bull., 30, 608611 (2007).

10) Hirai S., Yashiki T., Matsuzawa T., Mima H. Int. J. Pharm., 7, 317325 (1981).

11) Yamaoka K., Tanigawara Y., Nakagawa T., Uno T., J. PharmacobioDyn., 4, 879-885 (1981).

12) Hardy J. G., Lee S. W., Wilson C. G., J. Pharm. Pharmacol., 37, 294 297 (1985).

13) Hidalgo I. J., Raub T. J., Borchardt R. T., Gastroenterology, 96, 736749 (1989).

14) Grainger C. I., Greenwell L. L., Lockley D. J., Martin G. P., Forbes B., Pharm. Res., 23, 1482-1490 (2006).

15) Hussain A., Ahsan F., J. Drug Target., 14, 181-190 (2006). 